\title{
Announcements
}

INTERNATIONAL CONFERENCE ON APPLICATIONS OF SUPERCOMPUTERS IN ENGINEERING

Aachen (West Germany); July 19-21, 1987. Enquiries to:

Liz Newman

Conference Secretary

Computational Mechanics Institute

52 Henstead Road

SOUTHAMPTON SO1 2DD

England

(Tel. 0703 221398; telex 47388 Attn COMPMECH)

1987 SUMMER SIMULATION CONFERENCE

Montreal, Quebec (Canada); July 27-30, 1987. Enquiries to: SCS

P.O. Box 17900

SAN DIEGO

CA 92117

USA

(Tel. 619 277-3888)

SECOND INTERNATIONAL CONFERENCE ON APPLICATIONS OF ARTIFICIAL INTELLIGENCE IN ENGINEERING

Boston, Mass. (USA); August 4-7, 1987. Enquiries to Liz Newman, as above:

\section{THIRD INTERNATIONAL CONFERENCE ON} ADVANCES IN PRODUCTION MANAGEMENT SYSTEMS

Winnipeg, Manitoba (Canada); August 11-14, 1987. Enquiries to:

\author{
APMS-87 Conference \\ 356 Engineering Building \\ Dept. of Mech. \& Prod. Engineering \\ University of Manitoba \\ WINNIPEG \\ Manitoba R3T 2N2 \\ Canada
}

\section{FIRST EUROPEAN SOFTWARE ENGINEERING \\ CONFERENCE}

Strasbourg (France); September 8-11, 1987. Enquiries to: AFCET

156, Boulevard Péreire

75017 PARIS

France

(Tel. 1476624 19: telex Eurtel 290163 Code 235)

\section{CONFERENCE ON COMPUTER SIMULATION}

Bangor, North Wales (U.K.); September 9-11, 1987. Enquiries to:
Dr R. N. Zobe
Dept. of Computer Science
University of Manchester
MANCHESTER M13 9PL
England

\section{INTERNATIONAL CONFERENCE ON RELIABILITY AND ROBUSTNESS OF ENGINEERING SOFTWARE} Como (Italy); September 23-25, 1987. Enquiries to $\mathrm{Liz}$ Newman, as above

\section{CONFERENCE ON DATABASES AND KNOWLEDGE \\ BASES}

Nice (France); September 28-29, 1987. Enquiries to AFCET, as above

\author{
FOURTH FABTECH INTERNATIONAL CONFERENCE \\ AND EXHIBITION \\ Chicago, Illinois (USA); September 28-October 1, 1987. \\ Enquiries to: \\ SME Public Relations \\ One SME Drive \\ P.O. Box 930 \\ DEARBORN \\ Michigan 48121 \\ USA \\ (Tel. 313 271-1500; telex 297742 SME UR (VIA RCA)
}

\section{FIFTH INTERNATIONAL IMEKO SYMPOSIUM ON} TECHNICAL DIAGNOSTICS

Paderborn (West Germany); October 7-9, 1987. Enquiries to: Prof. Dr. Ing. D. Barschdorff

Elektrische Messtechnik

Universität-Gesamthochschule

Warburger Str. 100

D-4790 PADERBORN

West Germany

(Tel. 05251/603022)

THIRD INTERNATIONAL CONFERENCE ON SPACE INDUSTRY..SPACETECH '87

London (U.K.); October 8-9, 1987. Enquiries to: ONLINE International Ltd

Pinner Green House

Ash Hill Drive

PINNER HA5 2AE

Middlesex

England

(Tel. 01868 4466; telex 923498 ONLINE G)

\section{COMPUTER GRAPHICS EXHIBITION AND}

CONFERENCE

London (U.K.); October 13-15, 1987. Enquiries to ONLINE, as above 
THIRD INTERNATIONAL CONFERENCE ON ADVANCED ROBOTICS 'Towards third generation robotics'

Versailles (France); October 13-15, 1987. Enquiries to:

INRIA

Domaine de Voluceau

Rocquencourt

BP 105

78153 LE CHESNAY Cedex

France

(Tel. 33139635600 ; telex INRIA 697033 F)

\section{CONFERENCE AND EXHIBITION ON AUTOMATED} INTEGRATED FACTORY

Detroit, Michigan (USA); November 9-12, 1987. Enquiries to SME, as above

\section{SIXTH CONGRESS AND EXHIBITION ON PATTERN} RECOGNITION AND ARTIFICIAL INTELLIGENCE

Antibes (France); November 18-20, 1987. Enquiries to INRIA, as above

\section{CONFERENCE AND EXHIBITION ON SYSTEM SECURITY}

London (U.K.); November 17-19, 1987. Enquiries to ONLINE, as above

\section{CONFERENCE AND EXHIBITION ON COMPUTERS} IN THE CITY

London (U.K.); November 17-19, 1987. Enquiries to ONLINE, as above

\section{INTERNATIONAL EXHIBITION FOR MEASURING}

AND AUTOMATIC CONTROL.. REGITECH '87

Lenigrad (USSR); November 19-26, 1987. Enquiries to: NOWEA International GmbH

Postfach 320203

Stockumer Kirchstrasse 61

D-4000 DÜSSELDORF 30

West Germany

(Tel. 02114560 02; telex 8588351 now d)

SEVENTEENTH ISATA CONFERENCE (CIM \& MAP)

Munich (West Germany); October 26-28, 1987. Enquiries to:

Dr John I. Soliman

Chairman, ISATA Co-ordinating Committee

42 Lloyd Park Avenue

CROYDON CRO 5SB

Surrey

England

(Tel. 016867026 ; telex 28905 Monref 2592)

\section{INTERNATIONAL SYMPOSIUM ON THE}

TECHNOLOGIES FOR OPTOELECTRONICS

Cannes (France); November 16-20, 1987. Enquiries to: SPIE

P.O. Box 10

BELLINGHAM

WA $98227-0010$

USA

(Tel. 2066763290 ; telex 46 7053)

\section{CONFERENCE ON VOICE PROCESSING}

London (U.K.); December 2-3, 1987. Enquiries to ONLINE, as above

\section{FOURTH ANNUAL SYMPOSIUM ON}

MICROPROCESSOR-BASED PROTECTION SYSTEMS

London (U.K.); December 10, 1987. Enquiries to:

Rosamund da Gama

Manager, Conference Division

The Institute of Measurement and Control

87 Gower Street

LONDON WC1E

England

(Tel. 01387 4949)

EIGHTH INTERNATIONAL CONFERENCE ON COMPUTING METHODS IN APPLIED SCIENCES AND ENGINEERING

Versailles (France); December 14-18, 1987. Enquiries to INRIA, as above

INTERNATIONAL WORKING CONFERENCE ON THE SOCIAL IMPLICATIONS OF ROBOTICS AND ADVANCED INDUSTRIAL AUTOMATION

Eilat (Israel); December 14-17, 1987. Enquiries to: Conference on Robotics

c/o International Ltd

P.O. Box 29313

TEL AVIV 61292

Israel

(Tel. 03 654541; telex 33544) 